\title{
Efficient Hardware Code Generation for FPGAs
}

\author{
ZHI GUO, WALID NAJJAR; and BETUL BUYUKKURT \\ University of California, Riverside
}

The wider acceptance of FPGAs as a computing device requires a higher level of programming abstraction. ROCCC is an optimizing $\mathrm{C}$ to HDL compiler. We describe the code generation approach in ROCCC. The smart buffer is a component that reuses input data between adjacent iterations. It significantly improves the performance of the circuit and simplifies loop control. The ROCCCgenerated datapath can execute one loop iteration per clock cycle when there is no loop dependency or there is only scalar recurrence variable dependency. ROCCC's approach to supporting while-loops operating on scalars makes the compiler able to move scalar iterative computation into hardware.

Categories and Subject Descriptors: B.5 [Register-transfer-level Implementation]; B.5.2 [Design Aids]; C.3 [Signal Processing Systems]

General Terms: Design, Languages, Performance

Additional Key Words and Phrases: Reconfigurable computing, high-level synthesis, data reuse, FPGA, VHDL

\section{ACM Reference Format:}

Guo, Z., Najjar, W., and Buyukkurt, B. 2008. Efficient hardware code generation for FPGAs. ACM. Trans. Architec. Code Optim. 5, 1, Article 6 (May 2008), 26 pages. DOI = 10.1145/1369396.1369402 http://doi.acm.org/10.1145/1369396.1369402

\section{INTRODUCTION}

Continued increases in integrated circuit chip capacity have led to the recent introduction of the configurable system-on-a-chip (CSoC), which has one or more microprocessors integrated with a field-programmable gate array (FPGA) and memory blocks on a single chip [Altera Corp. 2005; Trisend Corp. 2004; Xilinx Corp. 2004]. The capabilities of these platforms span a wide range, having the flexibility of software along with the efficiency of hardware. They combine on one chip the sequential and the spatial computation models: The sequential parts of an application execute on the microprocessor, while the compute-intensive

Authors' address: Zhi Guo, Walid Najjar, and Betul Buyukkurt, University of California, Riverside, Riverside, California 92502; email: zguo@cs.ucr.edu.

Permission to make digital or hard copies part or all of this work for personal or classroom use is granted without fee provided that copies are not made or distributed for profit or direct commercial advantage and that copies show this notice on the first page or initial screen of a display along with the full citation. Copyrights for components of this work owned by others than ACM must be honored. Abstracting with credit is permitted. To copy otherwise, to republish, to post on servers, to redistribute to lists, or to use any component of this work in other works requires prior specific permission and/or a fee. Permissions may be requested from the Publications Dept., ACM, Inc., 2 Penn Plaza, Suite 701, New York, NY 10121-0701 USA, fax +1 (212) 869-0481, or permissions@acm.org. (C) 2008 ACM 1544-3566/2008/05-ART6 \$5.00 DOI 10.1145/1369396.1369402 http://doi.acm.org/ $10.1145 / 1369396.1369402$

ACM Transactions on Architecture and Code Optimization, Vol. 5, No. 1, Article 6, Publication date: May 2008. 
computations, which typically consist of parallel loops, are mapped as circuits on the FPGA. In other words, the FPGA acts as a configurable hardware accelerator or coprocessor to the microprocessor itself. Speedups ranging from $10 \times$ to $1000 \times$ over microprocessors have been reported for a variety of applications, including image and signal processing, DNA string matching, and protein folding [Berkeley Design Technology 2004; Keane et al. 2004; Chen et al. 2004]. Such speedups are the result of two main factors: large-scale parallelism and customized circuits. Applications, such as signal, image, and video processing exhibit very large amounts of parallelism, so mapping such a computation to a circuit can drastically improve its efficiency as compared to a traditional microprocessor. These factors have been described and quantitatively evaluated [Guo et al. 2004a].

The main problem standing in the way of wider acceptance of CSoC platforms is their programmability. Currently, application developers must have extensive hardware expertise, in addition to their application area expertise, to develop efficient designs that can fully exploit the potential of CSoC. Designing and mapping large applications onto FPGAs is a long and tedious task that involves a large amount of low-level design in a hardware description language (HDL). To bring CSoCs into the mainstream, tools are needed that would map applications expressed in a high-level language to an efficient circuit in HDL.

Optimizing compilers for traditional processors have benefited from several decades of extensive research that has led to extremely powerful tools. Similarly, electronic design automation (EDA) tools have also benefited from several decades of research and development leading to powerful tools that can translate VHDL and Verilog code, and, recently, SystemC [SystemC Consortium 2005] code, into efficient circuits. However, little work has been done to combine these two approaches. Several projects have implemented various types of HLL to HDL translations (GARP [Callahan et al. 2000], Streams-C [Gokhale et al. 2000], SA-C [Najjar et al. 2003], DEFACTO [Diniz et al. 2001], SPARK [SPARK project 2005], Handel-C [Handel-C Language 2004] etc.). Two papers [Frigo et al. 2001; Guo et al. 2003] have reported on the performance gap between compiler-generated VHDL and hand-crafted VHDL for medium-size codes. In both cases, it is reported that the hand-crafted versions ran twice as fast.

ROCCC (riverside optimizing configurable computing compiler) is a secondgeneration compilation tool targeting $\mathrm{CSoC}$ leveraging on our prior experience with SAC [Najjar et al. 2003]. It takes high-level code, such as C or FORTRAN, as input and generates RTL VHDL code for FPGAs. One of its objectives is to bridge the above-described performance gap. Compiling to FPGAs is challenging. Traditional CPUs, including VLIW, have a fixed hardware structure with predetermined resources, such as ALUs and registers, and a protocol to use these resources-the instruction-set architecture (ISA). FPGAs, on the other hand, are completely amorphous. The task of an FPGA compiler is to generate both the data path and the sequence of operations (control flow). This lack of architectural structure, however, presents a number of advantages:

1. The parallelism is very high and limited only by the size of the FPGA device or by the data memory bandwidth in and out of the FPGA. Therefore, 


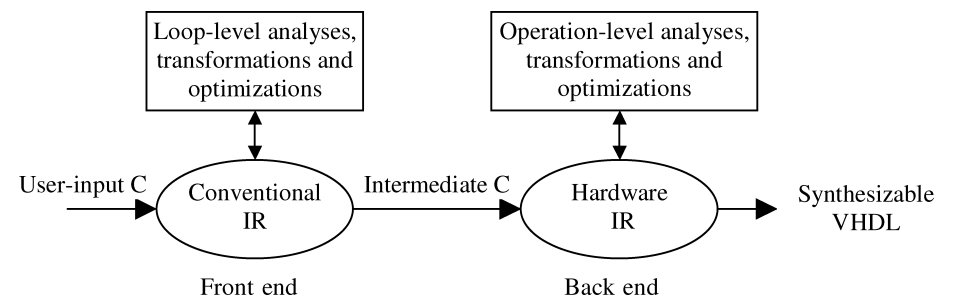

Fig. 1. ROCCC system overview.

loop transformations that can maximize the parallelism are of paramount importance.

2. On-chip storage can be configured at will: registers are created by the compiler and distributed throughout the data path where needed, thereby increasing data reuse and reducing accesses to memory.

3. Circuit customization: the data path and sequence controller are tailored to the specific computation being mapped to hardware. Examples include pipelining and customized data path bit width.

In this paper, we focus on the last two points. In previous work [Guo et al. 2005], we described our approach for the generation of pipelined data paths for do-all for-loops. In our implementation, a new instance of the loop body is started each cycle in the data path. In order to sustain that throughput, we must have a storage mechanism that is capable of feeding the data path with the required data. In [Guo et al. 2004b], we introduced the smart buffer, an interface between on-chip memory and the loop data path, whose objective is to minimize the number of data refetches from memory.

This paper complements and extends our previous work [Guo et al. 2004b; 2005]. In addition to the code generation of parallel (for) loops, we present and demonstrate, through examples, our approach for sequential (while) loops' data-path generation. We describe a novel and improved implementation of the smart buffer [Guo et al. 2004b] that (1) supports loops having multiple input and output arrays and (2) is more area and clock-cycle efficient. The smart buffer, by reusing the previously fetched data, makes the most of the memory bandwidth and minimizes the stall cycles of the pipelined data path.

The rest of this paper is organized as follows: the next section presents an overview of the ROCCC compiler framework. Section 3 presents the data-path generation for do-all for and while-loops. Results for each loop type are given in the subsections where the corresponding data-path generation is described. Section 4 introduces the smart buffer. Section 5 discusses related work. Section 6 concludes the paper.

\section{ROCCC OVERVIEW}

ROCCC is built on the SUIF2 [Aigner et al.; SUIF Compiler System 2005] and Machine-SUIF [Machine-SUIF 2005; Smith and Holloway, Machine-SUIF 2005] platforms. Figure 1 shows ROCCC's system overview. It compiles code written in $\mathrm{C} / \mathrm{C}++$ or Fortran to VHDL code for mapping onto the FPGA fabric 


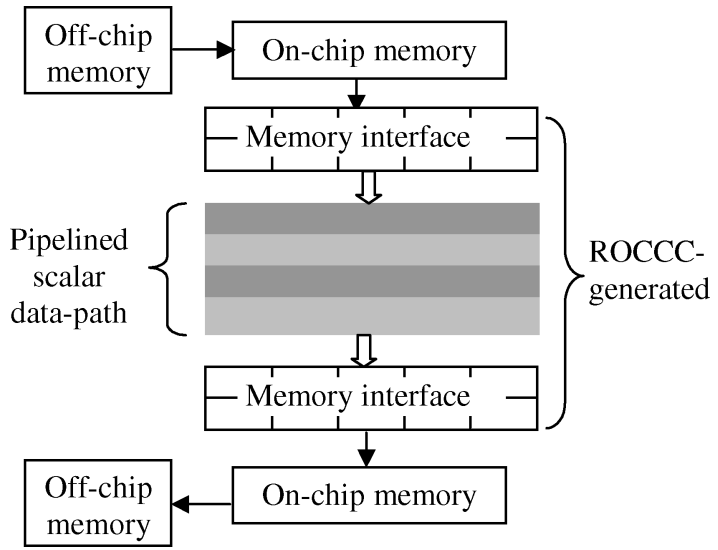

Fig. 2. Machine model of ROCCC.

of a CSoC device. In the execution model underlying ROCCC, sequential computations are carried out on the microprocessor in the CSoC, while the computeintensive code segments are mapped onto the FPGA. These typically consist of loop nests, most often parallel loops, operating on large arrays or streams of data. The front end of ROCCC performs a very extensive set of loop analysis and transformations aimed at maximizing parallelism and minimizing the area. The transformations include loop unrolling and strip mining, loop fusion, and common subexpression elimination across multiple loop iterations. ${ }^{1}$ Most of the information needed to design high-level components, such as controllers and address generators, is extracted from this level's IRs.

The machine model of ROCCC, shown in Figure 2, consists of on-chip memories (BRAM on the Xilinx architecture), memory interfaces, and a pipelined scalar data path. The scalar data path accesses memory only through memory interfaces. The compiler performs scalar replacement transformation at the front end. Figure 3 shows a simple example. The compiler converts the code segment in Figure 3a into the segment in Figure 3b, separating memory accesses from computations. Figure $3 \mathrm{c}$ is the hardware implementation of the highlighted segment (the scalar data path).

ROCCC uses Machine-SUIF virtual machine (SUIFvm) [Holloway and Smith 2002a] intermediate representation as the back-end IR. The original SUIFvm assemblylike instructions, by themselves, cannot completely cover HDLs' functionality. For example, the statement $(\mathrm{sum}=\mathrm{sum}+\mathrm{x}$ ) in a loop body will result in a loop-carried dependency. On FPGAs, this dependency can be simply removed by inserting a feedback variable between two adjacent pipeline stages. However, the SUIFvm assemblylike instruction set does not have an equivalent operation to describe this behavior. To compensate for this lack, ROCCC performs high-level data flow analysis at the front end and the analysis information is transferred through predefined macros to assist the back-end hardware generation.

\footnotetext{
${ }^{1}$ These transformations are beyond the scope of this paper. 


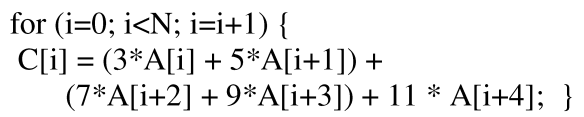

(a) A 5-tap FIR in original $\mathrm{C}$ code

for $(\mathrm{i}=0 ; \mathrm{i}<17 ; \mathrm{i}=\mathrm{i}+1)\{$

$\mathrm{A} 0=\mathrm{A}[\mathrm{i}] ; \quad \mathrm{A} 1=\mathrm{A}[\mathrm{i}+1] ; \quad \mathrm{A} 2=\mathrm{A}[\mathrm{i}+2]$

$\mathrm{A} 3=\mathrm{A}[\mathrm{i}+3] ; \quad \mathrm{A} 4=\mathrm{A}[\mathrm{i}+4] ;$

Tmp $0=3 * \mathrm{~A} 0+5 * \mathrm{~A} 1+7 * \mathrm{~A} 2$

$\mathrm{C}[\mathrm{i}]=\mathrm{Tmp} 0 ; \quad\}$ $+9 * \mathrm{~A} 3+11 * \mathrm{~A} 4$

(b) The FIR after scalar replacement.

The highlighted region has only scalars and corresponds to the data path.

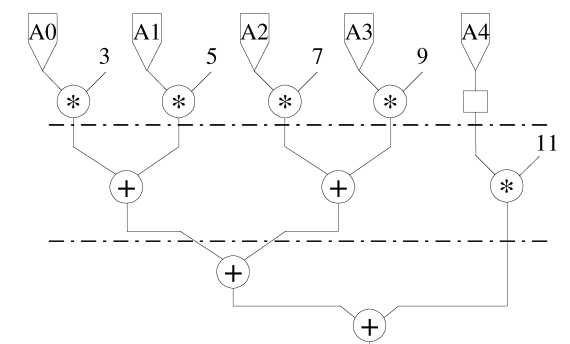

(c) The pipelined data path

Each pipeline stage is one instantiation of an iteration and three iterations can be executed at the same time.

Fig. 3. A 5-tap FIR and the corresponding data path.

The front-end's optimized output is fed into Machine-SUIF to generate lowlevel IRs. Machine-SUIF is an infrastructure for constructing the back end of a compiler. We modify Machine-SUIF's virtual machine (SUIFvm) intermediate representation (IR) [Holloway and Smith 2002a] to build our data flow. All arithmetic opcodes in SUIFvm have corresponding functionality in IEEE 1076.3 VHDL, with the exception of division. Machine-SUIF's existing passes, like the control-flow graph (CFG) library [Holloway and Smith 2002b], data-flow analysis library [Holloway and Dimock 2002], and static-single assignment (SSA) library [Holloway 2002], provide useful optimization and analysis tools for our compilation system. After applying SSA, control-flow graph information is visible and every virtual register is assigned only once.

After back-end analyses and optimizations, the compiler generates VHDL code. We rely on commercial tools, such as Synplicity [2005], to synthesize the VHDL code generated by our compiler.

We constrain the source code that will be translated to hardware, which is loop nests, as follows: no pointers, no break or continue statements, and all memory addresses must be resolvable at compile time.

\section{DATA-PATH GENERATION}

In terms of executing a loop in high-level languages, such as $\mathrm{C}$, on reconfigurable fabric, FPGA's most significant advantages are its lack of predesigned structure and its capacity for parallelism, both at the loop and instruction levels. One of its main weaknesses is the inefficiency of the automatic generation of custom control logic. For parallel loops, the main objectives of our data-path code generation are:

1. Exploiting potential loop-level and instruction-level parallelism.

2. Simple control of the generated data path.

3. Pipelining to achieve maximum throughput.

This section describes our approach for the compiler's data-path generation. 


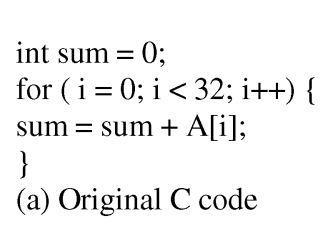

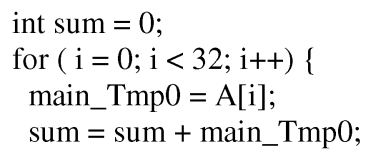

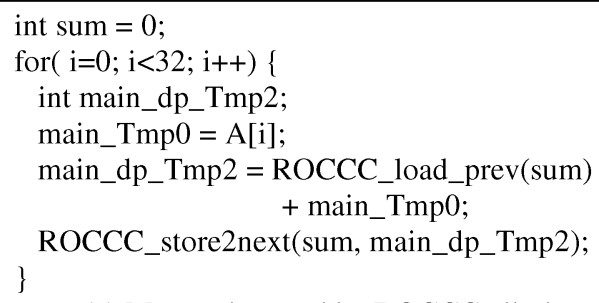

Fig. 4. The $\mathrm{C}$ code and the data-path of an accumulator.

\subsection{Preparation Passes}

After applying scalar replacement and front-end data-flow analysis, the program, such as the code shown in Figure 4c, is passed to Machine-SUIF. ROCCC performs circuit-level optimizations and eventually generates the data path in a modified version of the Machine-SUIF virtual machine intermediate representation. Figure $4 \mathrm{~b}$ shows an accumulator after applying scalar replacement in C. The variable sum is detected as a recurrence variable and will be a feedback signal in hardware. Figure $4 \mathrm{c}$ shows the resultant code segment in $\mathrm{C}$. The macros ROCCC_load_prev() and ROCCC_store2next() implement the recurrence.

Macros are converted into ROCCC-specific opcodes. For example, ROCCC_load_prev() and ROCCC_store2next() in Figure 4c are converted into instructions with opcodes $L P R$ (load previous) and $S N X$ (store next), respectively. $L P R$ is implemented as a feedback wire and $S N X$ is implemented as a register. This pair of instructions duplicates the variable of the present iteration to the next one and removes the loop-carried dependency. Lookup-table macros are also converted into corresponding LUT instructions.

\subsection{Building the Data Path}

Each instruction that goes to hardware is assigned a location in the data path. We add a new field, $[n]$, as shown in Equation (1), into Machine-SUIF IR to record the location of each arithmetic, logic, or register-copying instruction in the data path. We call this location the execution level. The higher level an instruction is located, the earlier it is executed.

$$
[n][m] \quad \text { add } \quad \$ v r 1 . s 32<-\$ v r 2 . s 32, \quad \$ v r 3 . s 32
$$

The compiler groups the instructions in each node into different execution levels to exploit instruction-(operation) level parallelism. Instructions at the same level are executed simultaneously. Additional mov instructions are added, where needed, as passthrough nodes. Each instruction's location in the data 
path satisfies the following requirements:

-If an instruction's source operand(s) is the live-in operand of this node, the instruction must be at the top level of the data path. If an instruction's destination operand is the live-out operand of this node, the instruction must be at the bottom level of the data-path.

-An instruction's source operands are the destination operands of the instructions one level higher.

-If a live-in operand is also in the live-out operand set, it is copied down to the bottom level.

- Mux nodes are added to implement if-conversion. Latch nodes are added to copy live operands from a branch-node's preceding node down to their succeeding node. Alternative branches of the data path have the same numberof levels.

At this point, every level of the data-flow graph corresponds to the instantiation of one loop iteration. Superfluous mov instructions areeliminated by the synthesis tool.

ROCCC automatically places latches to pipeline a data path. Each execution level is marked as either latched or unlatched, according to the estimated sum of the signal propagation delay from the most recently latched level, and the special timing requirement of some instructions. Another field, $[m]$, shown in Equation (1), is added to record the latch level of an instruction. At a given execution level, all the instructions of that level are either latched or unlatched. All the operations between two latched levels are synthesized asone combinational circuit. Every latched level corresponds to one pipeline stage and has a delay of one cycle. A parameterized controller is generatedto clock the pipeline.

ROCCC generates one VHDL component for each CFG node that goes to hardware. In a node, every virtual register is single assigned and is converted into wires in hardware. Arithmetic, logic, and copying instructions become combinational or sequential VHDL statements, according to whether they are latched or not. A LUT instruction invokes an instantiation of a lookup table component. If the lookup table is a preexisting one, such as trigonometric or logarithmic function, the compiler automatically inserts the relevant values. Otherwise, the user provides the table entries, for example, to describe a probability distribution function. In this case, the compiler instantiates the lookup table as a regular ROM IP core unit in the VHDL code.

By adding more data types in Machine-SUIF, ROCCC supports any signed and unsigned integer and fixed-point type and size. The compiler infers the inner signals' bit size automatically from the arithmetic operations.

\subsection{Comparison with Xilinx IP Cores}

Two previous works have compared compiler generated to handwritten VHDL codes for SA-C [Guo et al. 2003] and StreamsC [Frigo et al. 2001]. In both cases, it was shown, independently and on different examples, that the handwritten VHDL achieved a clock frequency one half as large as the compiler-generated codes. Achieving a comparable clock rate is one of the objectives of ROCCC. 
Table I. A Comparison of Hardware Performance from Xilinx IPs and ROCCC-Generated VHDL Code

\begin{tabular}{|c|c|c|c|c|c|c|c|c|}
\hline & \multicolumn{3}{|c|}{ Xilinx IP } & \multicolumn{3}{|c|}{ ROCCC } & \multirow[b]{2}{*}{$\%$ Clock } & \multirow[b]{2}{*}{$\%$ Area } \\
\hline Example & $\begin{array}{c}\text { Clock } \\
(\mathrm{MHz})\end{array}$ & $\begin{array}{l}\text { Delay } \\
(\mathrm{cycl})\end{array}$ & $\begin{array}{c}\text { Area } \\
\text { (slice) }\end{array}$ & $\begin{array}{l}\text { Clock } \\
(\mathrm{MHz})\end{array}$ & $\begin{array}{l}\text { Delay } \\
(\mathrm{cycl})\end{array}$ & $\begin{array}{c}\text { Area } \\
\text { (slice) }\end{array}$ & & \\
\hline bit_correlator & 212 & 1 & 9 & 144 & 2 & 19 & 0.679 & 2.11 \\
\hline mul_acc & 238 & 1 & 18 & 238 & 1 & 59 & 1.00 & 3.28 \\
\hline udiv & 216 & 11 & 144 & 272 & 25 & 495 & 1.26 & 3.44 \\
\hline square root & 167 & 25 & 585 & 220 & 37 & 1199 & 1.32 & 2.05 \\
\hline $\cos$ & 170 & 1 & 150 & 170 & 1 & 150 & 1.00 & 1.00 \\
\hline Arbitrary LUT & 170 & 1 & 549 & 170 & 1 & 549 & 1.00 & 1.00 \\
\hline FIR & 185 & 17 & 270 & 194 & 1 & 293 & 1.05 & 1.09 \\
\hline DCT & 181 & 20 & 412 & 133 & 2 & 724 & 0.735 & 1.76 \\
\hline DWT* & 104 & 1 & 1464 & 101 & 3 & 2415 & 0.971 & 1.65 \\
\hline & & & & & & Average: & 1.001 & 1.93 \\
\hline
\end{tabular}

${ }^{*}$ DWT code is handwritten.

We, therefore, compare the hardware performance generated from Xilinx IP cores and ROCCC-generated VHDL code. We use Xilinx ISE 5.1i and IP core 5.1i. All the Xilinx IP cores and ROCCC-generated VHDL code are synthesized targeting a Xilinx Virtex-II xc2v2000-5 FPGA. All the benchmarks in Table I are from Xilinx IP core, except the DWT engine that we wrote. The input and output variables of ROCCC equivalents have the same bit sizes as that of the IP cores.

Bit_correlator counts the number of bits of an 8-bit input data that are the same as of a constant mask. Mul_acc is a multiplier accumulator, whose input variables are a pair of 12-bit data. Udiv is an 8-bit unsigned divider. Square_root calculates a 24-bit data's square root. Cos's input is 10 bit; its output is 16 bit. The arbitrary $L U T$, whose content can be defined by users in a text file before synthesis, has the same port size as that of cos. FIR is two five-tap 8-bit constant coefficient finite impulse response filters, whose bus sizes are 16-bit. $D C T$ is a one-dimensional 8-data discrete cosine transform. The input data size and output data size are 8 and 19 bit, respectively. For Xilinx IP FIR and $D C T$, multiplications with constants are implemented using the distributed arithmetic technique, which performs multiplication with lookup-table based schemes. Therefore, we set the synthesis option "multiplier style" as "LUT" for the ROCCC-generated DCT and FIR. The second through the fourth column of Table I show Xilinx IP cores' clock rate, delay in clock cycle, and device utilization, respectively. The fifth through the seventh column show ROCCC's corresponding performance. \%Clock is the percentage difference in clock rate of ROCCC-generated VHDL compared to Xilinx IP. \%Area is the percentage difference in area of ROCCC-generated VHDL compared to Xilinx IP.

Bit-correlator, udiv, and square root consist of a large number of bitmanipulation operations, which, for the C language, is not well suited to express. This is the major source of the performance difference. Xilinx mul_acc IP has a control input signal nd (new data) whose Boolean value true indicates the present data is valid. In $\mathrm{C}$ code, we describe the equivalent behavior using an ifelse statement whose condition evaluates the Boolean input $n d$, requiring extra 
nodes and latches to be added to support the alternative branch, consuming extra area. (We also tried changing this $\mathrm{C}$ code simply by multiplying $n d$ with the new input data instead of using the if-else statement. Although one more multiplier was used, the overall area and clock rate performance was better than that listed in Table I. Obviously, that is not a compile-level optimization, but it does show one of the advantages of high-level synthesis: ease of algorithm level optimizations). In terms of lookup tables, the ROCCC-generated VHDL code instantiates Xilinx IP cores, so they have exactly the same performance. In Xilinx Virtex-II, 10-bit input-16-bit output cos / sin lookup table stores only half wave, which is one of the reasons that this cos/sin lookup table utilizes less area compared with the arbitrary ROM lookup table with the same port size. Fir operates on an array: basically, a five-data element window slides over the one-dimensional array, and ROCCC generates a smart buffer to reuse the previous input data. The FIR's data path consists of multipliers and adders with no branch. ROCCC fits this type of algorithm and gets comparable performance with IP cores. The IP core has several handshaking signals. The ROCCC-generated FIR does not have those handshaking signals, since its data communication method with outside is known at compile time. Like FIR, DCT has high computational density and no branch. The throughput of Xilinx DCT IP is one output data element per clock cycle, while ROCCC's throughput is eight output data elements per clock cycle. Therefore, although ROCCC-generated DCT runs at a lower speed (73.5\%), the overall throughput of the ROCCC-generated circuit is higher. Both ROCCC DCT and Xilinx IP DCT exploit the symmetry within the cosine coefficients. The last row in Table I shows an implementation of a two-dimensional $(5,3)$ wavelet transform engine, which is the standard lossless JPEG2000 compression transform. This DWT engine includes the address generator, smart buffer, and data path. The ROCCC-generated circuit is compared with a handwritten one.

We derive the bit-width information based only on port size and opcodes. More aggressive bit-width narrowing transformations, performed by users and/or the compiler, may further reduce device area utilization.

\subsection{The Data-Path Generation and the Control of a While-Loop}

A while-loop is an inherently sequential construct that is not usually considered a candidate for mapping to hardware. However, often signal- and imageprocessing algorithms have a while-loop nested in a parallel for-loop or vice versa. The internal structure of a von Neumann processor is tailored for the execution of sequential codes, and can, therefore, easily support the execution of a while-loop. A spatial implementation, however, lacks a program counter, so the compiler must generate a simple, yet efficient, customized control structure for each while-loop. Figure 5a shows an example in $\mathrm{C}$, in which the main computational burden is in the multiplication that squares $x[i]$. In order to put the whole for-loop in hardware, we also need to support the inner while-loop in hardware. While-loops usually cannot be unrolled and their implementation must support feedback of variables between iterations.

In Figure 5, nodes 2 and 3 correspond to the while-loop: at the bottom of node 2 , there is a branch instruction to assert whether the loop body, node 3 , should 


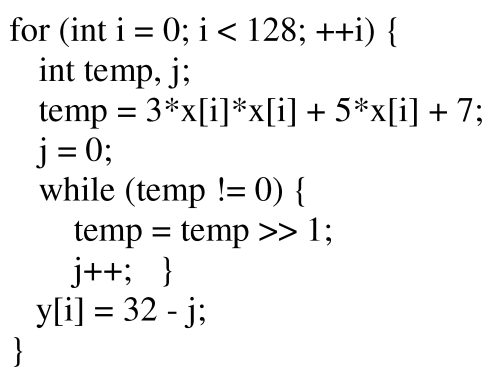

(a) The source code in C. We support while-loops to exploit parallelism in outer loops

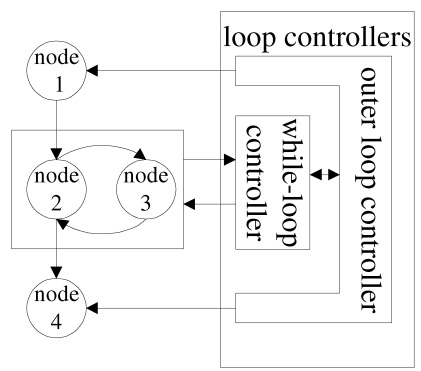

(b) The execution architecture. Node 2 is the header node.

Fig. 5. A while-loop embedded in a for-loop.

be executed or not. Node 2 is called the header node of a while-loop. The loop controller consists of two subcontrollers, the while-loop controller and the outer loop controller.

The controller first activates the while-loop's predecessor node(s), for example, node 1 in Figure 5b. In the predecessor nodes, multiple loop iterations are instantiated since each level corresponds to one iteration and we have assumed no loop dependency. Once the earliest iteration reaches the bottom of node 1, the controller halts node 1 and activates node 2 . The while-loop controller here does not activate the whole data path of node 2: rather, it activates the while-loop data path from top to bottom, stage by stage. The while-loop branch instruction, which is at the bottom level of node 2, generates the assertion loop_again signal to the while-loop controller. After the execution of the branch instruction in the header node, the controller evaluates the loop_again signal from the data path to determine whether to enter the loop body or the successor node following it. If the loop_again is set, the controller signals the while-loop body to execute, after which, the controller rewinds to execute the header node again. If, instead, the loop_again signal is clear, the controller halts the while-loop body and activates the while-loop's successor node as it did to the predecessor nodes. The loop controllers are written in synthesizable VHDL with heavy use of generics. These generics describe the length of the while-loop data path and that of the outer for-loop, the while-loop's location relative to the outer loop, and the location of the branch instruction of the while-loop.

The most significant difference between a while-loop data path and the rest of the outer for-loop data-path is that the former has to deal with feedback variables from the while-loop body nodes to the header node. In order to simplify the controller, ROCCC forces all feedback variables to be assigned in the bottom while-loop body node. Otherwise, the variable is copied down to the bottom node. We create a new instruction in the Machine-SUIF virtual machine instruction set, FOI (feedback or initialization). The instruction format of FOI is as follows.

$$
\text { foi } \$ \text { vr1.s32 <- \$vr2.s32, \$vr3.s32 }
$$


Table II. Description and Source Code of the While Loop Examples

\begin{tabular}{|c|c|c|}
\hline & Description & Source code \\
\hline GCD_ifelse & $\begin{array}{l}\text { The segment calculates the } \\
\text { greatest common divisor } \\
\text { using if-else statement. }\end{array}$ & $\begin{array}{l}\mathrm{a}=\mathrm{x} ; \quad \mathrm{b}=\mathrm{y} \\
\text { while }(\mathrm{a} !=\mathrm{b})\{ \\
\quad \text { if }(\mathrm{a}>\mathrm{b}) \mathrm{a}=\mathrm{a}-\mathrm{b} \\
\text { else } \mathrm{b}=\mathrm{b}-\mathrm{a} ;\} \\
\text { gcd }=\mathrm{a}\end{array}$ \\
\hline GCD_minmax & $\begin{array}{l}\text { The segment calculates the } \\
\text { greatest common divisor } \\
\text { using } \min () \text { and } \max () \\
\text { macros. }\end{array}$ & $\begin{array}{l}\mathrm{a}=\mathrm{x} ; \quad \mathrm{b}=\mathrm{y} ; \quad \min =\mathrm{x} ; \quad \max =\mathrm{y} ; \\
\text { while }(\mathrm{a} !=\mathrm{b})\{ \\
\quad \min =\text { ROCCC_min }(\mathrm{a}, \mathrm{b}) \\
\max =\text { ROCCC_max }(\mathrm{a}, \mathrm{b}) \\
\quad \mathrm{a}=\min ; \quad \mathrm{b}=\max -\min ;\} \\
\text { gcd = min } ;\end{array}$ \\
\hline Dif_equi & $\begin{array}{l}\text { The algorithm numerically } \\
\text { obtains } \mathrm{y} \text { in equation } \\
\frac{d^{2} y}{d x^{2}}+3 x \frac{d y}{d x}+3 y=0 \\
\text { where } \mathrm{x} \text { starts from } \mathrm{x} \_ \text {in to a } \\
\text { with step } \mathrm{dx} \text {. }\end{array}$ & $\begin{array}{l}\mathrm{u}=\mathrm{u} \text { _in } ; \quad \mathrm{x}=\mathrm{x} \text { in } ; \quad \mathrm{y}=\mathrm{y} \text { in; } \\
\mathrm{u} 1=\mathrm{u} ; \quad \mathrm{x} 1=\mathrm{x} ; \quad \mathrm{y} 1=\mathrm{y} ; \\
\text { while }(\mathrm{x}<\mathrm{a})\{ \\
\quad \mathrm{x} 1=\mathrm{x}+\mathrm{dx} ; \quad \mathrm{u} 1=\mathrm{u}-3^{*} \mathrm{dx} *(\mathrm{u} * \mathrm{x}+\mathrm{y}) \\
\mathrm{y} 1=\mathrm{y}+\mathrm{u} * \mathrm{dx} ; \quad \mathrm{x}=\mathrm{x} 1 ; \quad \mathrm{y}=\mathrm{y} 1 ; \quad \mathrm{u}=\mathrm{u} 1 ;\} \\
\text { x_out }=\mathrm{x} 1 ; \\
\text { y_out }=\mathrm{y} 1 ; \\
\text { u_out }=\mathrm{u} 1 ;\end{array}$ \\
\hline Integ_equi & $\begin{array}{l}\text { The algorithm numerically } \\
\text { solves equation } \\
\int_{a}^{y} \cos ^{2} x d x=\text { const }\end{array}$ & $\begin{array}{l}\text { sum }=0 ; \quad \mathrm{x}=\mathrm{a} \\
\text { while }(\text { sum }<\text { const })\{ \\
\text { temp }=\cos (\mathrm{x}) ; \quad \text { sum }=\text { sum }+ \text { temp*temp } \\
\mathrm{x}=\mathrm{x}+1 ;\} \\
\mathrm{y}=\mathrm{x}\end{array}$ \\
\hline Bisect-root & $\begin{array}{l}\text { Using bisection method, the } \\
\text { algorithm finds the root of } \\
\text { equation } x^{2}-60 x+500=0 \\
\text { in the range of }[\mathrm{a}, \mathrm{b}] .\end{array}$ & 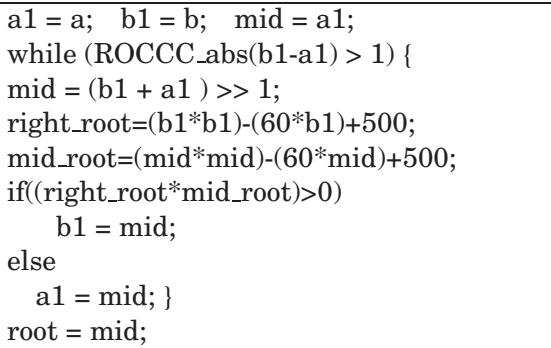 \\
\hline
\end{tabular}

This instruction only appears on the top of a while-loop's header node. When executed, it evaluates signal FOI $i$, which comes from the while-loop controller. If $F O I i$ is clear, the destination operand equals the first source operand; otherwise, the destination operand equals the second source operand. The hardware implementation of instruction $F O I$ is a multiplexer. The first time a header node is executed, the controller signals the FOI instructions (multiplexers in hardware) to select the upper node's output variables. From then on, the controller signals the FOI instructions to select the feedback variables. The FOI instruction is also used to copy constant variables from a while-loop's predecessor node to the while-loop body.

\subsection{While-Loop Examples}

Table II lists five examples having a while-loop inside a for-loop. Figure 6 shows the IR and data path of the Bisect_root example. 
Node: 2 (the while loop's header node) top latch level: 8 , bottom latch level: 7 [8] foi \$vr231.s32<- \$vr230.s32,\$vr37.s32

[8] foi $\$ v$ r229.s32<- \$vr228.s32,\$vr36.s32

[8] foi $\$ v r 227 . s 32<-\$ v r 226 . s 32, \$ v r 35 . s 32$

[8] foi $\$ v r 225 . s 32<-\$ v r 224 . s 32, \$ v r 34 . s 32$

[7] mov \$vr220.s32<- \$vr225.s32

[7] mov \$vr221.s32<- \$vr227.s32

[7] mov \$vr222.s32<- \$vr229.s32

[7] mov \$vr223.s32<- \$vr231.s32

[7] ble \$vr231.s32,1,df_bisection._bisectionTmp1

Node: 3 (one of the while loop's body nodes) top latch level: 6, bottom latch level: 5

[0] add \$vr67.s32<- \$vr221.s32,\$vr220.s32

[0] mul \$vr78.s32<- \$vr221.s32,\$vr221.s32

[0] mul $\$$ vr80.s32<-60,\$vr221.s32

[0] mov \$vr220.s32<- \$vr220.s32

[0] mov \$vr221.s32<- \$vr221.s32

[0] asr \$vr232.s32<- \$vr67.s32,1

[0] sub $\$$ vr81.s32<- \$vr78.s32,\$vr80.s32

[6] mov $\$$ vr70.s32<- \$vr232.s32

[6] add $\$ v$ r84.s32<- $\$$ vr81.s32,500

[6] mul \$vr85.s32<- \$vr232.s32,\$vr232.s32

[6] mul \$vr87.s32<-60,\$vr232.s32

(a) IR. We are only presenting the IR of node 2 and node 3 . The foi instructions are highlighted. The instructions with zero latch level correspond to combinational logic.

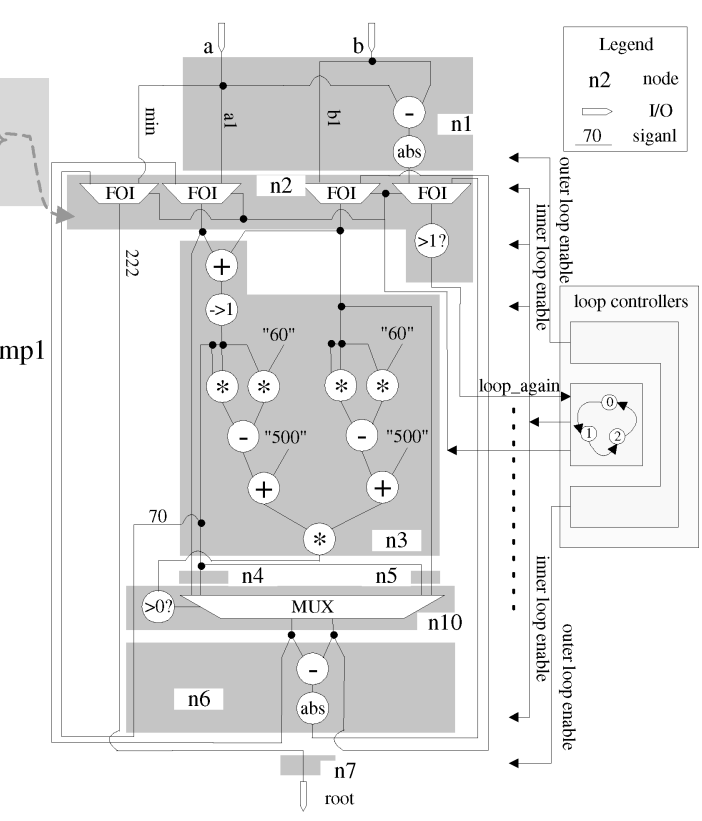

(b) The data path. Node $\mathrm{n} 2$ is the whileloop's header node.

Fig. 6. IR and data path of bisect root.

Table III. Hardware Performance of the While-Loop Examples

\begin{tabular}{|l|c|c|c|c|}
\hline & Area (slices) & Multpl $(18 \times 18)$ & Clock $(\mathrm{MHz})$ & Cycle Per Iteration \\
\hline GCD_ifelse & 275 & 0 & 69.9 & 6 \\
\hline GCD_minmax & 191 & 0 & 76.7 & 3 \\
\hline Dif_equi & 370 & 12 & 40.9 & 5 \\
\hline Integ_equi & 140 & 1 & 56.5 & 4 \\
\hline Bisect_root & 590 & 15 & 51.1 & 8 \\
\hline
\end{tabular}

Table III shows the place-and-route results and execution results from simulation. The bit size of Integ_equi is 10 bit and all the other examples' signals are 32 bit. The last column is the number of cycles per iteration. In GCD ifelse the if-else statement is converted by ROCCC: both branches execute and the predicate selects the outcome. GCD_minmax, on the other hand, utilizes predefined macros to pick min and max values. These macros, instantiated as RTL VHDL function calls in the generated VHDL code, are more efficient. GCD_minmax's generated data path is shorter and takes fewer clock cycles compared with $G C D$ ifelse with no pipeline idle cycles.

\section{INPUT DATA REUSE}

Signal, image, and video processing are among the primary target applications of reconfigurable computing. Window operators are frequently used in these 


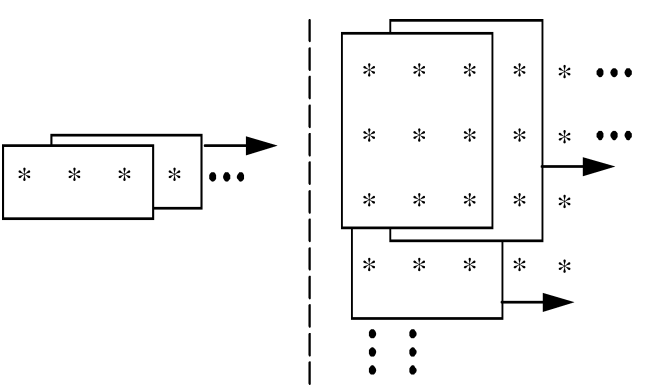

(a) $1 \mathrm{D}$ and $2 \mathrm{D}$ window operators

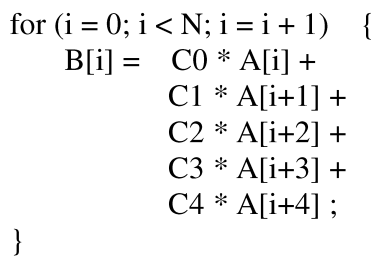

(b) A 5-tap FIR in C

Fig. 7. One- and two-dimensional window operation examples.

applications. Examples include FIR (finite impulse response) filters in signal processing, edge detectors, erosion/dilation operators, and texture measures in image/video processing. All these window operators have similar calculation patterns-a loop or a loop nest operates on a window of data (in other word, a pixel/sample and its neighbors), while the window slides over an array, as shown in Figure 7a. In most cases, these window operators are do-all for-loops and their data paths can be generated using the approach presented in the previous section.

However, in order to take advantage of the high-throughput of the data path, input data has to be organized and fed in efficiently. Figure 7b shows a five-tap FIR filter example code in C. B[i] is the filter's output and $\mathrm{A}[\mathrm{i}]$ is the input. $\mathrm{C} 0$, $\mathrm{C} 1, \mathrm{C} 2, \mathrm{C} 3$, and $\mathrm{C} 4$ are the filter's constant coefficients. In the previous section, we already showed that the throughput of the compiler-generated data path is one iteration per clock cycle. However, if a reconfigurable computing compiler performs a straightforward hardware generation, the functional unit would need to access all five input data values in the current window. This would require a large amount of memory bandwidth and involve pipeline bubbles in the data path.

Balancing computation with I/O has been considered a critical factor of the overall performance for quite some time [Kung 1982]. When a high-density computation is performed on a large amount of input data, as the case in window operations, data $\mathrm{I} / \mathrm{O}$ often dominates the overall computation performance. For instance, for the window operations reported in [Guo et al. 2004a], the general-purpose CPU performed $64 \mathrm{X}$ to $112 \mathrm{X}$ more load operations per pixel than a hand-crafted circuit on an FPGA. Therefore, in order to achieve high performance, a reconfigurable computing compiler needs to generate smart hardware in HDL to reduce the memory bandwidth pressure by exploiting data reuse whenever possible. We call this piece of synthesizable HDL module smart buffer. The compiler must implement the smart buffer tailored for the applications, and schedule the buffer's reads and writes.

\subsection{Execution Architecture and Code Analysis}

In window-based operations, the input and output arrays (or streams) are separate and, therefore, there is no loop-carried dependency on a single array. 


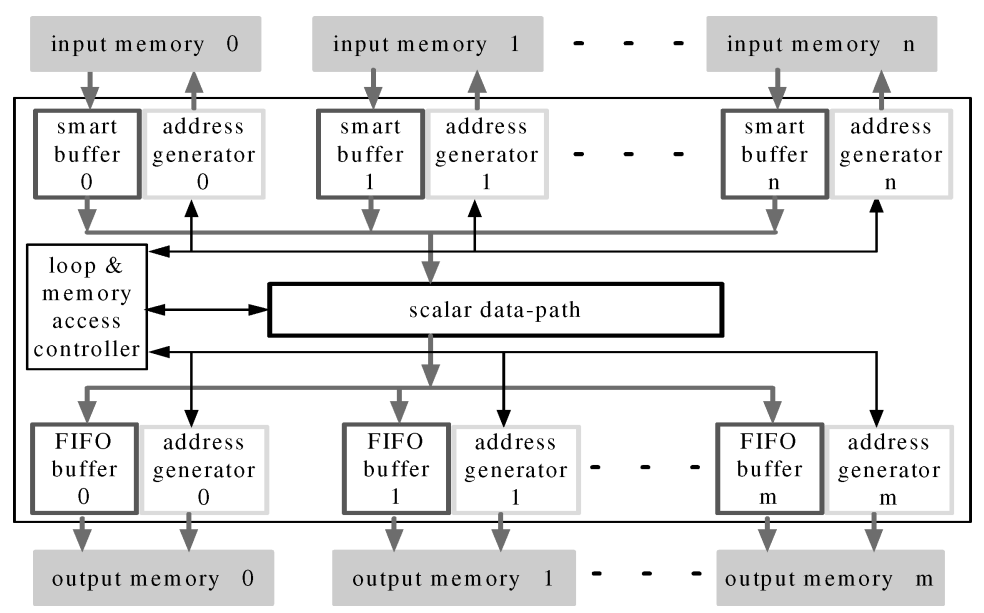

Fig. 8. Overall window operator execution architecture. Memory accessing is decoupled with calculation.

An execution architecture of window operators is shown in Figure 8. The ROCCC framework is not board specific in that it does not assume a preset number of memory modules connected to the FPGA. The data communication engine between the on-chip block RAMs and the interchip data streams is not part of the ROCCC code generation. The loop has $n$ input and $m$ output arrays. If $n$ is equal to one, the compiler generates a single-mode smart buffer. Otherwise, it generates multimode smart buffers. Each input/output array of a loop corresponds to an input/output memory. One of the most important characteristics of window operations is that the compiler can decouple the memory accesses from the computations and thereby can maximize data reuse. Every input or output memory is connected to a compiler-generated smart buffer, or a compiler-generated FIFO (first-in-first-out) buffer, respectively. The compiler performs scalar replacement on the loop body in the front end. Therefore, in Figure 8, the data path, by itself, does not access memory directly. The address generators are VHDL libraries. The input address generator generates memory load addresses and feeds the addresses to the on-chip block memory. The smart buffer gets the input data streams from the block memory, exploits data reuse, and makes the data of the current window available to the data path. In other words, the smart buffer collects/reuses the input data of one iteration and exports them into the data path at the same time. In this way, the computation and memory access are decoupled. The write buffer collects the results from the data path and presents it to the output memory. The output address generator generates memory store addresses.

A one-dimensional example algorithm and a two-dimensional example algorithm are shown in Figure $7 \mathrm{~b}$ and Figure 9, respectively. We use them to explain how the ROCCC compiler generates smart buffer. For the algorithm in Figure 9, the output pixel is the difference between the output of the low-pass filter (upper part in Figure 9) and the pixel of the reference image. Figure 10 gives the unrolled $\mathrm{C}$ code before undergoing scalar replacement. The compiler 


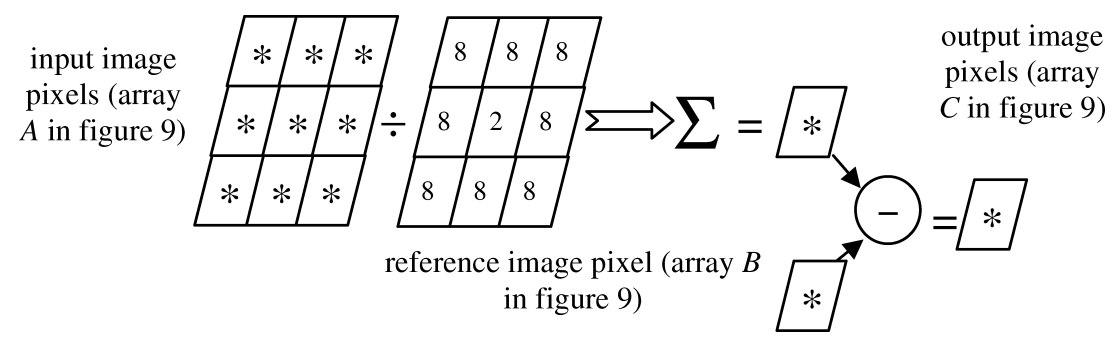

Fig. 9. $3 \times 3$ Motion detection window. The upper part is a 2D low-pass filter.

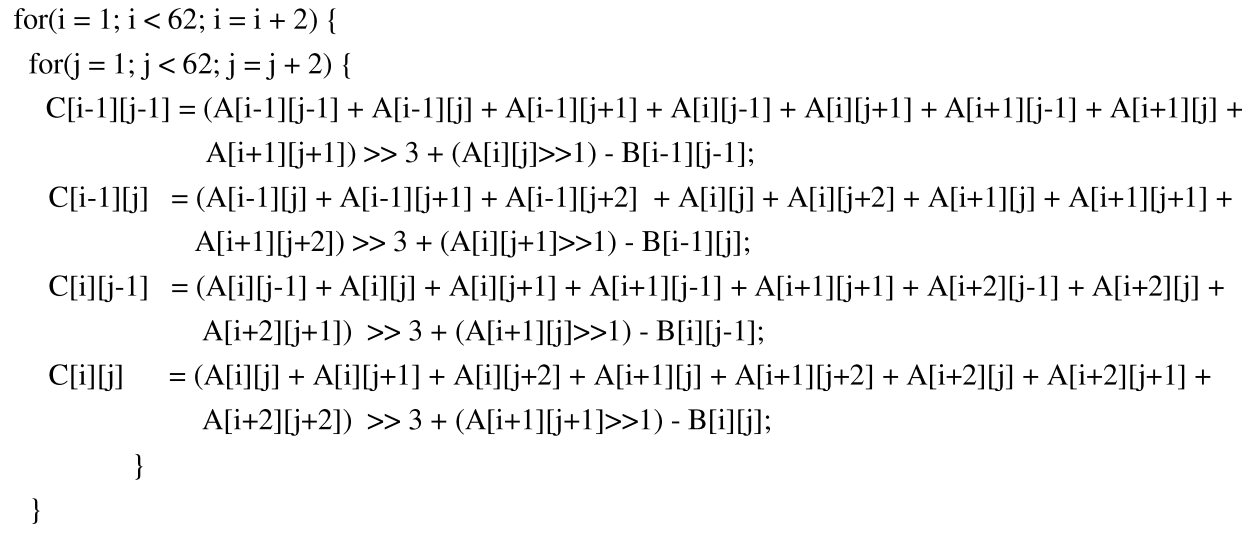

Fig. 10. Motion detection $\mathrm{C}$ code $2 \times 2$ unrolled loop for the algorithm in Figure 9 . Array $A$ and $B$ correspond to input memory banks and array $C$ correspond to output memory bank.

walks through all the memory references in the SUIF IR and confirms that there is no array being both read and written. The compiler also checks the following constraints.

1. Loop counters are assigned and updated only in the loop statements.

2. Each loop counter determines the memory address calculation in only one dimension.

\subsection{VHDL Code Generation}

Figure 11 shows the registers of the FIR filter [Figure 7b]'s one-dimensional smart buffer. In this figure, we assume that the memory I/O bus is 32 bit and the data width is 8 bit. In order to fully utilize memory bandwidth, we unroll the for-loop four times. The unrolled for-loop body has four copies of the original loop body. Now each iteration reads eight contiguous array elements, but the stride between two iterations is four $(\mathrm{i}=\mathrm{i}+4)$. The smart buffer has eight elements. In Figure 11, parts (1) through (3) show the smart buffer's status from clock cycle one through four. At cycle two, the smart buffer gets the first four data. At cycle three, the smart buffer collects the eight data elements needed for the current iteration and initiatively exports them to the data path. Notice also that at cycle three, the left-most four elements are killed to reclaim space for 
6:16 - Z. Guo et al.

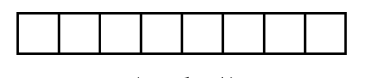

(cycle 1)

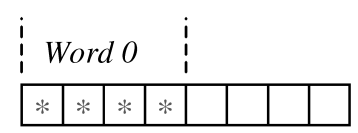

(cycle 2)

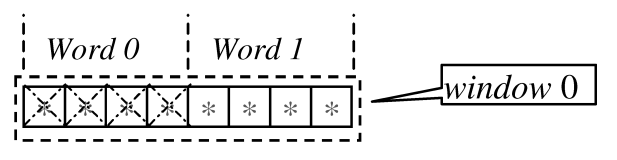

(cycle 3)

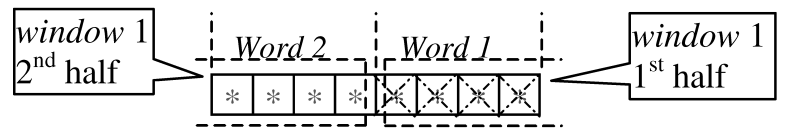

(cycle 4)

Fig. 11. The smart buffer of the four-time-unrolled five-tap FIR filter.

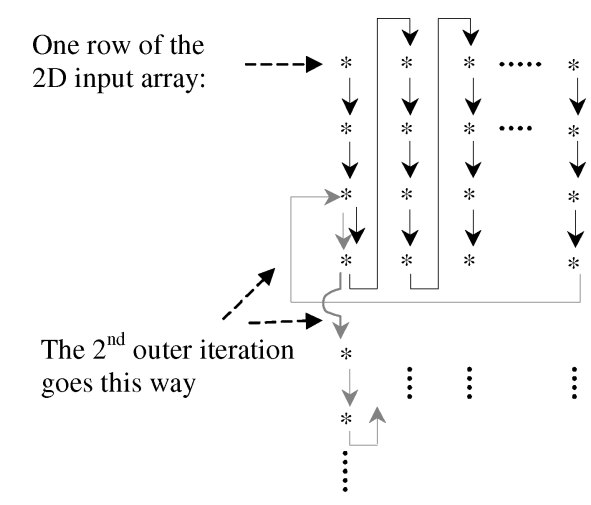

Fig. 12. The reference address order of array $A$ in figure 10 .

word 2. At cycle four, window 1 starts from the right-most four elements in the buffer. The ROCCC compiler generates both the registers and the logic to schedule the registers' action.

In this subsection we present ROCCC's approach to the generation of efficient VHDL code for the smart buffers and related components. The goal is to minimize runtime control calculation and maximize input data reuse.

4.2.1 Address Generation. Window operations have one or more windows sliding over one or more arrays. Both the read and the write array addresses are known are compile time. We also assume that the on-chip memory read latency is known. ${ }^{2}$

${ }^{2}$ This is not an unusual assumption in FPGA design.

ACM Transactions on Architecture and Code Optimization, Vol. 5, No. 1, Article 6, Publication date: May 2008. 


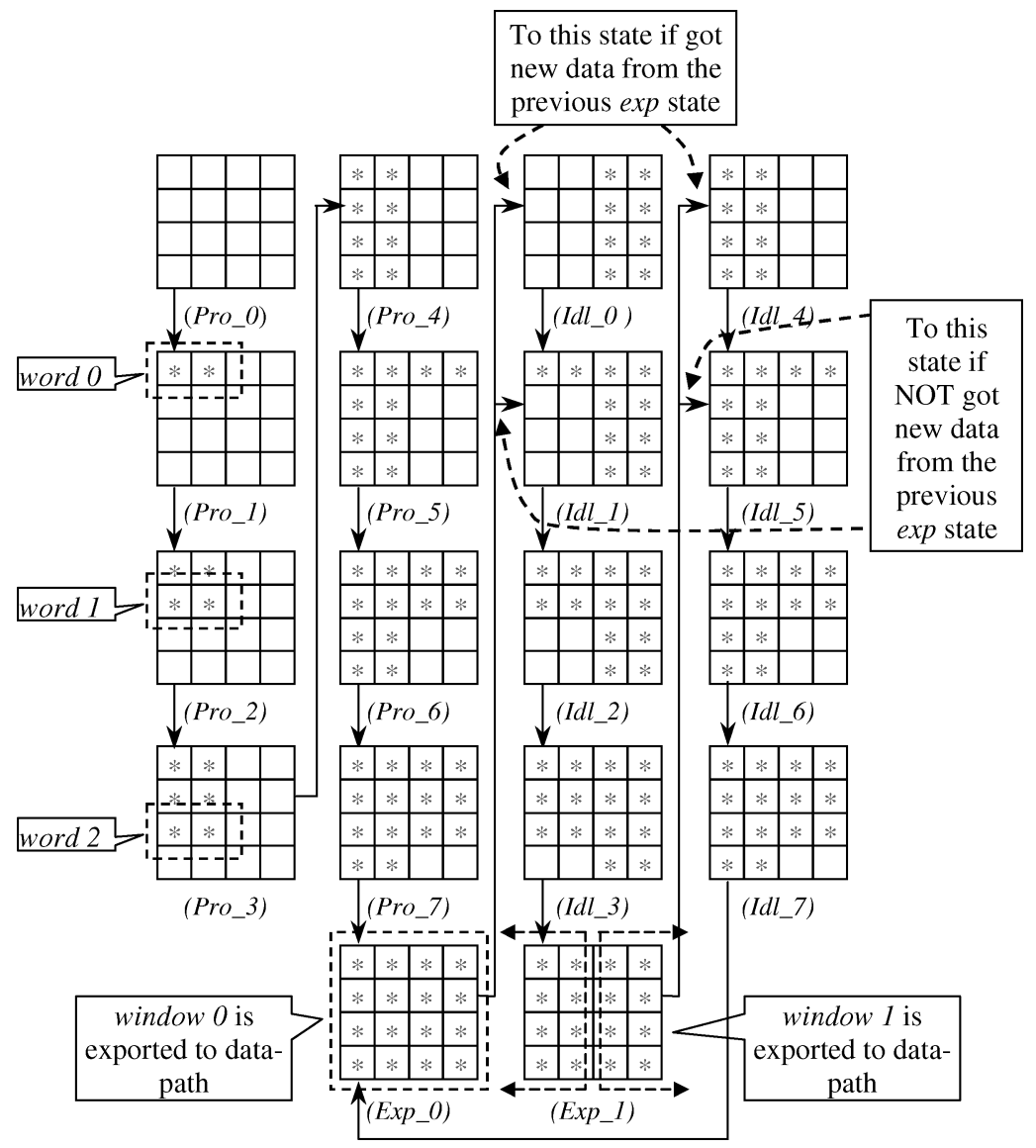

Fig. 13. FSM Status of a two-dimensional smart buffer.

Consider the code in Figure 10 as an example. According to the memory load references and the loop unrolling parameters, the following parameters are known at compile time:

1. Starting and ending addresses

2. The number of clock cycles between two consecutive memory accesses

3. The unrolled window's size

4. The unrolled window's strides in each dimension

5. The array's row size

6. The starting address-difference between two adjacent outer-loop iterations.

In Figure 8, each smart buffer has an address generator to generate the loading address stream to the input memory bank. These address generators are parameterized finite state machines (FSMs) in VHDL. Figure 12 shows the loading address stream of the two-dimensional array $A$ in Figure 10. An address generator does not produce all the loading addresses of the corresponding array for the current iteration; rather, it only produces the new data's addresses. The 


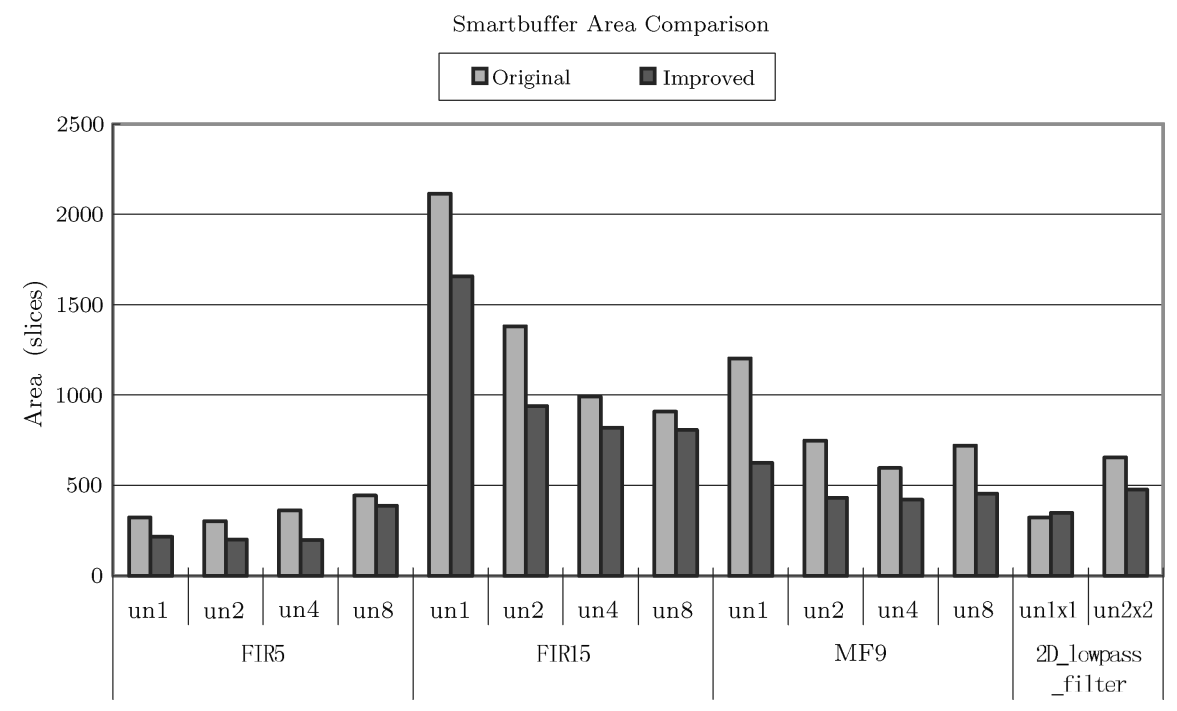

Fig. 14. Smart buffer area comparison between original and improved architectures.

remaining recently loaded data are already in the smart buffer. In Figure 12, the input data needed by each outer-loop iteration are loaded only once. However, there are overlaps between adjacent outer iterations, since we cannot afford a smart buffer to hold whole rows of data. Notice that the first inner-loop iteration of each outer-loop iteration might read more columns of data compared with other inner-loop iterations, because, for the first iteration, all the loads are new. We call these extra new columns warm-up columns.

In the case of the data path producing output data at a higher rate than the loading rate from the input memory, and the output memory bandwidth is limited, the loading-address generator has to slow down its address stream to balance the I/O throughput by inserting extra idle cycles after each iteration's loading. All these analyses can be done at compile time and thereby simplify the controller.

The VHDL generics of an address generator include:

- The number of warmup-columns (the number of warmup-data for onedimensional address generators).

- The number of new columns per iteration (the number of new data per iteration for one-dimensional address generators).

- The number of cycles to halt between iterations. This parameter might be zero. These halt cycles are inserted to balance the I/O throughput between input and output memories.

- The number of loads per outer loop, the number of data per row, and the address difference between two adjacent outer-loop iterations' upper-left-most loads. These parameters only apply to two-dimensional address generators.

With the above parameters, each input memory's address generator is able to generate the address stream of the current iteration's new data and each 
output memory's address generator can generate the corresponding storing addresses for the current iteration's output data. Once the generation of the current iteration's address stream is done, all address generators check if the loop and memory access controller has issued the next iteration, and act accordingly. For two-dimensional-array cases, the above parameters also ensure that the loading-address generators are able to determine when a new outer-loop iteration starts, and wait until then to flush the smart buffers. Notice that all loading-address generators have to flush the smart buffers though the loopand memory-access controller. The latter flushes all the smart buffers at the same cycle to maintain the synchronicity of the data path's input data.

4.2.2 Smart Buffer Generation. An input array's smart buffer accomplishes the following tasks:

-Collecting the new input data for the current iteration.

-If the new input data are needed by future iterations, storing them to buffer registers whose contents are expired.

-Exporting the window data to the data path when they are all ready. For multiple-input array loops, the multimode smart buffers hold the window data until the controller signals it to release. This way all smart buffers are synchronized. A single-input array loop's smart buffer pushes the window data into the data path immediately.

A smart buffer is implemented in VHDL at compile time. It consists of registers to store the data and a finite state machine (FSM). The FSM traces which register is expired and would be overwritten by new data, determines when a window of data is ready to export, and manages the counterpart relationship between the buffer registers and the data path's input variables.

We use the 2D array $A$ in Figure 10 as an example. Figure 13 shows the status of array A's smart buffer at different clock cycles. Each iteration of the nested loop loads a $4 \times 4$ window. We assume the memory bus is twice the width of the pixel bit size, so each memory load reads in two pixels. In this example, the window's row size is an integer multiple of the bus load (one row is two bus loads). If this is not the case, for example, each window row has five pixels, we round the smart buffer row size to an integer multiple of the bus size, which makes the smart buffer row size six pixels (three bus loads). Each FSM state is assigned one of the three states:

-Prolog state: The computation is in a warm-up state of the first iteration of loops working on one-dimensional arrays or of the first inner-loop iteration of every outer-loop iteration of loops working on two-dimensional arrays. The smart buffers are collecting data to form the first window.

-Export state: In this state, a window of data is ready. If the loop has only one input array, the smart buffer's FSM initiates the export of the window data to the data path and keeps going to the next state. For multimode smart buffers, the FSM stays in this state until the loop controller signals it to release. 
-Idle state: In this state, the smart buffer is waiting for the new data that have not been loaded in previous iterations. Once all the new data have arrived, the smart buffer goes to the export_state.

Generally, for a two-dimensional smart buffer, the row size of the buffer is the smallest integer multiple of the bus-size that makes the row size larger than the sliding window's row size. The smart buffer's column size is equal to the height of the sliding window. For a one-dimensional smart buffer, the column size is always one.

As shown in Figure 13, the state machine initially starts from state Pro_0. Once it gets export state Exp_0, window_0 is exported to the input port of the data path. Since array $A$ is one of the two input arrays in Figure 10, the FSM stays in this state until the loop controller signals it to go to the next iteration. If at Exp_0 state the smart buffer gets another new bus of data, which would only happen in single-input-array cases, the state machine jumps to Idle_1. Otherwise it goes to Idle_0. Once the FSM leaves state Exp_0, the data elements in the left-most and the second left-most columns are killed to reclaim the registers for new data. In state Exp_1, the smart buffer exports window_1. Notice that the first column of this window is the third column in the buffer. The compiler is aware of this relationship and implements it in the export states accordingly. The new data to state Idle_7 switches the state machine back to Exp_0. In fact, the last columns of idle states (Idle_4 through Idle_7) are identical to the last column of prolog states (Pro_4 through Pro_7).

A two-dimensional array's smart buffer is able to be flushed and start the FSM from the first prolog state.

The generated VHDL code, by itself, does not have the concepts of windows. The VHDL code only describes the logical and sequential relationship between signals/registers.

Implementing smart buffers using shift registers, while is an option, would require too many counters inside the smart buffer. There also would have to be a great deal of logic between the counters to control the smart buffer's import and export actions. Besides, for the multiple input-array case, the synchronization requirement increases the complexity of the logic. By using the FSM, the compiler's analysis effort simplifies the generated circuit, and the FSM efficiently tracks the smart buffer's status.

\subsection{Experimental Results}

We use the five benchmarks listed in Table IV in our experiments. These benchmarks are selected for the diversity of their numbers of input arrays, dimension of arrays and memory interface bandwidth.

Constant_FIR is a constant-coefficient finite-impulse-response (FIR) filter. Its source code is given in Figure 3. Array $A$ is the only input array, whose dimension is one. Variable FIR is a variable-coefficient FIR filter. Its coefficients are also variables stored in Array $B$. This example has two one-dimensional input arrays. Each iteration of Complex FIR produces a complex integer. The complex integer's real and imaginary part are alternately stored in Array $C$. 
Table IV. Synthesis and Simulation Results of Buffers in Five Examples ${ }^{a}$

\begin{tabular}{|c|c|c|c|c|c|c|}
\hline & & $\begin{array}{c}\text { Constant } \\
\text { FIR }\end{array}$ & $\begin{array}{c}\text { Variable } \\
\text { FIR } \\
\end{array}$ & $\begin{array}{c}\text { Complex } \\
\text { FIR }\end{array}$ & $\begin{array}{c}\text { 2D_lowpass } \\
\text { filter }\end{array}$ & $\begin{array}{c}\text { Motion } \\
\text { detection }\end{array}$ \\
\hline \multirow{4}{*}{ Input buffer A } & Area (slices) & 156 & 159 & 132 & 325 & 327 \\
\hline & \# of regs & 5 & 5 & 6 & 16 & 16 \\
\hline & \# of states & 14 & 14 & 8 & 18 & 18 \\
\hline & Bus size (bits) & 8 & 8 & 16 & 16 & 16 \\
\hline \multirow[t]{4}{*}{ Input buffer B } & Area (slices) & & 159 & & & 150 \\
\hline & \# of regs & & 5 & & & 4 \\
\hline & \# of states & & 14 & & & 4 \\
\hline & Bus size (bits) & & 8 & & & 16 \\
\hline \multirow[t]{4}{*}{ Output buffer C } & Area (slices) & 11 & 11 & 12 & 73 & 73 \\
\hline & \# of regs & 1 & 1 & 2 & 2 & 2 \\
\hline & \# of states & 1 & 1 & 2 & 2 & 2 \\
\hline & Bus size (bits) & 8 & 8 & 8 & 16 & 16 \\
\hline \multirow[t]{2}{*}{ Data-path } & Area (slices) & 43 & 5 mltpl & 99 & 144 & 164 \\
\hline & Bit size & 8 & 8 & 8 & 8 & 8 \\
\hline \multicolumn{2}{|c|}{ Overall area (slices) } & 210 & 329 & 243 & 542 & 714 \\
\hline \multicolumn{2}{|c|}{ Clock rate $(\mathrm{MHz})$} & 94 & 68 & 85 & 69 & 42 \\
\hline \multicolumn{2}{|c|}{ Execution time (cycles) } & 262 & 1019 & 260 & 5980 & 5986 \\
\hline \multicolumn{2}{|c|}{ Throughput (iteration/cycle) } & 0.96 & 0.25 & 0.48 & 0.16 & 0.16 \\
\hline
\end{tabular}

${ }^{a}$ The Total number of slices is 46,592 on the target FPGA chip.

Therefore, every iteration loads in two new data. The loop counter step of Complex FIR is two. 2 _lowpass filter is a $3 \times 3$ low-pass filter used in image processing. For each $3 \times 3$ window in the input image, the nine pixels are divided by the corresponding coefficients (they really are shifting operations). The output pixel is the summation of the division's nine results. We unroll the loop twice in both horizontal and vertical directions. Therefore, each iteration computes four of these $3 \times 3$ windows and produces a $2 \times 2$ output window. The input of $2 D$ _lowpass filter is a two-dimensional array. Motion_detection is the implementation of the code in Figure 10. Motion_detection's input windows are $4 \times 4$ and $2 \times 2$. These two windows come from the image array to be detected and the reference image array, respectively. There is no data reuse to the reference image array and the smart buffer of it is used only to decouple the data path with memory accessing. ROCCC supports multiple output arrays, although all these examples have only one output array. We do not show a multiple-output array example because writing to each array is an independent process and the implementation is a straightforward replication.

We use the Xilinx ISE 6.2.03i tool chain to do synthesis and place-and-route. The generated VHDL codes are simulated using ModelSim 5.8c. The target architecture of all synthesis is Xilinx XC2V8000-5, whose total number of slices is 46,592 . We set the synthesis option "multiplier style" as "LUT" for all constant multiplications.

In Table IV, Area is the number of slices obtained from place-and-route reports. The area of a buffer includes the area of the address generator associated with the buffer. \# of reg is the number of registers that the corresponding buffer uses to store the data. Each register is 8 bit, since all the data path variables of these five examples are 8 bit. \# of state is the number of states of the 
Table V. Smart Buffer Size and Synthesis Results of Unrolled Loops

\begin{tabular}{|l|c|c|c|c|c|c|c|c|}
\hline & \multicolumn{2}{|c|}{ Un1 } & \multicolumn{2}{c|}{ Un2 } & \multicolumn{2}{c|}{ Un4 } & \multicolumn{2}{c|}{ Un8 } \\
\cline { 2 - 10 } & $\begin{array}{c}\text { Buffer } \\
\text { size (pixel) }\end{array}$ & Slices & $\begin{array}{c}\text { Buffer } \\
\text { size (pixel) }\end{array}$ & Slices & $\begin{array}{c}\text { Buffer } \\
\text { size (pixel) }\end{array}$ & $\begin{array}{c}\text { Buffer } \\
\text { Slices }\end{array}$ & size (pixel) & Slices \\
\hline constant_FIR5 & 5 & 213 & 6 & 199 & 8 & 197 & 16 & 388 \\
\hline constant_FIR15 & 15 & 1657 & 16 & 938 & 20 & 817 & 24 & 806 \\
\hline moving_Filter9 & 9 & 623 & 10 & 431 & 12 & 421 & 16 & 453 \\
\hline 2D_lowpass_filter & 9 & 242 & & & 16 & 376 & & \\
\hline
\end{tabular}

buffer's FSM. Bus size is the number of bits of the bus between input/output memory and the corresponding buffer. Clock rate is the clock rate of the whole placed-and-routed circuit. The input data set size of all one-dimensional examples is 256 and the input data set size of all two-dimensional examples is $64 \times 64$. Execution time is the number of cycles obtained from the simulation waveforms.

Constant_FIR has only one input array, whose dimension is one. The input smart buffer automatically feeds its window data to the data path whenever they are ready, without waiting for synchronization. The loop controller keeps issuing new iterations. The total execution time is just a few cycles more than the input data set size. The area cost on the input buffer's address generator of constant FIR is more significant, compared with that on the registers (only five 8 -bit registers). Variable FIR has two input arrays. Both the input smart buffers and the loop controller have to spend extra clock cycles to do handshaking with each other, so the execution time is longer compared with Constant_FIR. For Complex FIR, we intentionally set the input bus size to be 16 bit and the output bus size to be 8 bit. Each memory load can read in two input data, which is what one iteration needs, since the loop counter step is two. However, the compiler detects that the output FIFO buffer needs two cycles to store two output data of one iteration, the real and the imaginary part. The compiler adds one halt cycle to the input smart buffer's address generator for each iteration and this extra halt cycle explains the fact that Complex FIR's total execution time is almost the same as that of Constant_FIR, though the number of iterations of Complex_FIR is only one-half of that of Constant_FIR. 2D_lowpass_filter has a two-dimensional array and the compile chooses the corresponding smart buffer and loop controller. In contrast, Motion_detection has two input arrays. Like the case of Variable_FIR, synchronizing between smart buffers and loop controller needs extra clock cycles. However, these cycles overlap with the intrinsic delay of two-dimensional address generators. This is the reason that Motion_detection's execution time is close to that of $2 D$ lowpass filter.

The window size of $2 D$ _lowpass_filter is $4 \times 4$. Every time the sliding window reaches the right of the image, it rewinds back to the left and starts from the second last row of the previous outer loop, since the outer loop counter's stride is now four. Therefore, by using the smart buffer, we only reread each pixel once. Without the smart buffer, each pixel would be read four times. Obviously, the more a loop is unrolled, the more memory loads can be saved. Four loop unrolling examples are shown in Table V. Constant_FIR15 is a 15-tap constant-coefficient finite-impulse-response filter. Moving filter9 is a nine-element moving 
average filter. We assume the data size is 16 bit. Buffer size is the number of the 16-bit storage units in the smart buffer. The benchmarks are unrolled 2,4 , and 8 times, with the exception of $2 D$ _lowpass_filter, which is unrolled four times $(2 \times 2)$. We also assume that the bus bandwidth scales up with unrolling. Notice that the buffer size of constant FIR5 on Un8 is 16, although the number of memory loads per iteration is 12 . The compiler rounds the buffer size to an integer multiple of the bus size, which is 8 data per bus for Un8. When the loop is unrolled, the buffer size increases, but it holds fewer distinct windows and, therefore, the cost of control logic decreases while the storage area increases. This explains why the total area of the smart buffer does not increase linearly with the amount of unrolling.

The smart buffer's control logic determines the location of the input data's destination buffer registers, schedules the output data export, and determines the proper connection between the buffer registers and their corresponding data path input ports. Notice that this connection varies for different iterations as the window slides. For example, the connection is different between Exp_0 and Exp_1 in Figure 13. In our original work [Guo et al. 2006b], all these control is fulfilled by combinational circuit. The compiler made less analysis effort, but generated a less efficient circuit. Compared with our previous work, the current smart buffer's FSM is more area efficient. The new approach requires more sophisticated compile-time analysis to build the FSM. The bar chart in Figure 14 depicts the smart buffer area comparison. With the exception of 2D_lowpass_filter(un1x1), the area savings of the new smart buffers range from 13 to $93 \%$, and are $43 \%$, on average.

\section{RELATED WORK}

Many projects, employing various approaches, have worked on translating high-level languages into hardware. SystemC [2005] is designed to provide roughly the same expressive functionality of VHDL or Verilog and is suitable for designing software-hardware synchronized systems. Handle-C [2004] is a low-level hardware/software construction language with $\mathrm{C}$ syntax, which supports behavioral descriptions and uses a CSP-style (communicating sequential processes) communication model. Both SystemC and Handle-C are timed languages.

GARP [Callaham et al. 2000]'s compiler is designed for the GARP reconfigurable architecture, and generates GARP configuration file instead of standard VHDL. GARP's memory interface consists of three configurable queues, the starting and ending addresses of which are configurable. The queues' reading actions can be stalled.

SA-C [Najjar et al. 2003] (single assignment C), is a single-assignment highlevel synthesizable language. Because of special constructs specific to SA-C (such as window constructs) and its functional nature, its compiler can easily exploit data reuse for window operations. SA-C uses preexisting parameterized VHDL library routines to perform code generation in a way that requires a number of control signals between components and thereby involves extra clock cycles and delay.

ACM Transactions on Architecture and Code Optimization, Vol. 5, No. 1, Article 6, Publication date: May 2008. 
Streams-C [Gokhale et al. 2000] relies on the CSP model for communication between processes, both hardware and software, and can meet relatively high-density control requirements. One-dimensional input data reuse can be manually implemented in the source code.

SPARK [2005] is another C to VHDL compiler, which takes a subset of C as input and outputs synthesizable VHDL. Its optimizations include code motion, variable renaming, etc. The transformations implemented in SPARK reduce the number of states in the controller FSM and the number of cycles in the longest path.

GARP, Streams-C, and SPARK do not support accesses to two-dimensional arrays, so image-processing applications, including video processing, must be mapped manually.

Phoenix project [2005] has implemented a compiler called CASH, which represents the input program using Pegasus, a data-flow intermediate representation. CASH targets its asynchronous hardware. SOMA [Ventataramani et al. 2005] is a synthesis framework for constructing memory access network architecture, and has been integrated into CASH. SOMA features the support to input specifications in which memory references cannot be statically disambiguated.

DEFACTO [Diniz et al. 2001] system takes C as input and generates VHDL code. In its generated circuit, memory units are connected to the data path through the memory channel to reuse input data. The memory channel architecture has its FIFO queue and a memory-scheduling controller.

One main difference between ROCCC and these research projects is that ROCCC performs aggressive input data reuse.

\section{CONCLUSION}

In this paper we have presented the code generation part of ROCCC, an open framework built on the SUIF platform that compiles C programs to VHDL for mapping reconfigurable devices. At the front end, the compiler performs highlevel data-flow analysis, as well as an extensive set of loop transformations. It transfers the analysis information through preserved macros. At the back end, the compiler explores low-level parallelism, pipelines the data path, and narrows the bit sizes of the inner signals.

When compiling parallel loops, the ROCCC compiler generates a pipelined data path in which each pipeline stage corresponds to one iteration, so that the throughput is one iteration per clock cycle. We present our approach to maintaining the same throughput when there is only scalar recurrence between iterations. ROCCC supports lookup tables through automatically instantiating preexisting lookup table IPs or ROM IPs. The synthesis result shows that ROCCC-generated circuits take around $2 \mathrm{x} \sim 3 \mathrm{x}$ the area, but run at a clock rate comparable to that of existing Xilinx IPs. In many cases, the throughput of ROCCC-generated code is higher than that of the original IP. As expected, ROCCC performs better on high computational density examples than on high control density ones. When compiling sequential (while) loops, the compiler pipelines the data path in a similar way, but only one iteration is executed at a 
time. We created a new instruction to select a while-loop body's input variables between the feedback variables from the bottom of the loop body and the initial variables from the loop's predecessor node.

For parallel loops, in order to make the most of data path's one-iterationper-cycle throughput, we present a new approach to the reuse of data when compiling operations that involve sliding windows. We describe the compiler's analysis and optimization of the memory accesses in the $\mathrm{C}$ code, and we propose a compile-time scheme that generates a smart buffer for storing all the fetched data elements. Based on the number of input arrays, the smart buffer is generated in either single mode or multimode. Using its internal FSM, the smart buffer keeps track of the input data stream, exports valid window data to data path for each iteration, and kills expired data to reclaim registers for new ones. The smart buffer minimizes the rate of data refetch from external memory and also simplifies the loop control. At compile time the compiler balances the I/O throughput by inserting halt clock cycles in the input memory address generators. This approach simplifies the loop controller and saves feedback handshake signals between input and output buffers.

\section{REFERENCES}

Aigner, G., Diwan, A., Heine, D. L., Lam, M. S., Moore, D. L., Murphy, B. R., and Sapuntzakis, C. 2000. An Overview of the SUIF2 Compiler Infrastructure. Computer Systems Laboratory, Stanford University, CA.

Altera CoRP. 2004. Excalibur: System-on-a-Programmable. http://www.altera.com.

Callahan, T. J., Hauser, J. R., and WawrzyneK, J. 2000. The Garp Architecture and C Compiler.

Berkeley Design Technology, Inc. http://www.bdti.com/articles/info_eet0207fpga.htm\#DSPEnhanced\%20FPGAs, 2004.

Chen, W., Kosmas, P., Leeser, M., and Rappaport C. 2004. An FPGA implementation of the twodimensional finite-difference time-domain (FDTD) algorithm. In International Symposium on Field-Programmable gate Arrays (FPGA) (Febr.), Monterrey, CA.

Diniz, P., Hall Park, M., J. Park, J., So, B., and H. Ziegler, H. 2001. Bridging the gap between compilation and synthesis in the DEFACTO System. In Proceedings of the 14th Workshop on Languages and Compilers for Parallel Computing Synthesis (LCPC) (Oct.).

Frigo, J., Gokhale, M., ANd Lavenier, D. 2001. Evaluation of the streams-C C-to-FPGA compiler: An applications perspective. In Proceedings of the 9th ACM/SIGDA International Symposium on Field Programmable Gate Arrays (FPGA), Monterey, CA.

Gokhale, M. B., Stone, J. M., Arnold, J., And Lalinowski, M. 2000. Stream-oriented FPGA computing in the streams-C high level language. In IEEE Symposium on FPGAs for Custom Computing Machines (FCCM).

Goldstein, S., et al. 2005. http://www.cs.cmu.edu/-phoenix/index.html.

Guo, Z., Suresh, D. C., ANd NAJJAR, W. A. 2003. Programmability and efficiency in reconfigurable computer systems. Workshop on Software Support for Reconfigurable Systems, held in conjunction with the International Conference of High-Performance Computer Architecture (HPCA) (Febr.), Anaheim, CA.

Guo, Z., NAJJAR, W., VAhid, F., AND Vissers K. 2004a. A quantitative analysis of the speedup factors of FPGAs over processors. In International Symposium on Field-Programmable Gate Arrays (FPGA) (Febr.) Monterrey, CA.

Guo, Z., BuyukKuRT A. B., AND NAJJAR, W. 2004b. Input data reuse in compiling window operations onto reconfigurable hardware. In Proceedings of the ACM Symposium on Languages, Compilers and Tools for Embedded Systems (LCTES) (June), Washington, DC.

Guo, Z., Buyukkurt, B., NAJJAR, W., AND Vissers, K. 2005. Optimized generation of datapath from C Codes for FPGAs. In International ACM / IEEE Design, Automation and Test in Europe Conference (DATE) (Mar.), Munich. 
Handel-C. Language Overview. 2004. Celoxica, Inc. http://www.celoxica.com.

Holloway, G. 2002. The Machine-SUIF Static Single Assignment Library. Division of Engineering and Applied Sciences, Harvard University.

Holloway, G. ANd Smith, M. D. 2002a. Machine-SUIF SUIFvm Library. Division of Engineering and Applied Sciences, Harvard University, Cambridge, MA.

Holloway, G. ANd Smith, M. D. 2002b. Machine-SUIF Control Flow Graph Library. Division of Engineering and Applied Sciences, Harvard University Cambridge, MA.

Holloway, G. ANd Dimock, A. 2002. The Machine-SUIF Bit-Vector Data-Flow-Analysis Library. Division of Engineering and Applied Sciences, Harvard University Cambridge, MA.

KeAne, J., Bradley, C., And Ebeling, C. 2004. A compiled accelerator for biological cell signaling simulations. In International Symposium Field-Programmable Gate Arrays (FPGA) (Febr.), Monterrey, CA.

KunG, H. T. 1982. Why systolic architectures? IEEE Comput. 15, 1 (Jan.), 37-46.

MACHINE-SUIF. 2005. http://www.eecs.harvard.edu/hube/research/machsuif.html.

NajJar, W., Böhm, B., Draper, B., Hammes, J., Rinker, R., Beveridge, R., Chawathe, M., and Ross C. 2003. From algorithms to hardware-A high-level language abstraction for reconfigurable computing. IEEE Computer (Aug.).

SUIF Compiler System. 2005. http://suif.stanford.edu.

Smith, M. D. ANd Holloway, G. 2001. An Introduction to machine SUIF and its Portable Libraries for Analysis and Optimization. Division of Engineering and Applied Sciences, Harvard University. Cambridge, MA.

SPARK PROJECT. 2005. http://mesl.ucsd.edu/spark/.

SYstemC Consortium. 2005. http://www.systemc.org.

SYNPLICITY, Inc. 2005. http://www.synplicity.com/2005.

Triscend Corporation. 2004. Triscend A7 Configurable System on a Chip Family. http://www. triscend.com/products/a7.htm.

XILINX CorP. 2004. IBM and Xilinx Team. http://www.xilinx.com/prs_rls/ibmpartner.htm.

Venkataramani, G., BJerregaard, T., Chelcea, T., and Goldstein, S. C. 2005. SOMA: A tool for synthesizing and optimizing memory accesses in ASICs,. In International Conference on Hardware / Software Codesign and System Synthesis (CODES+ISSS) (Sept.), Jersey City, NJ,

Received February 2005; revised January 2006 and September 2006; accepted September 2007 